\title{
Adjuvant Therapy of Osteosarcoma-A Phase II Trial
}

\section{Southwest Oncology Group Study 9139}

\author{
Mark M. Zalupski, M.D. ${ }^{1}$ \\ Cathryn Rankin, M.s. ${ }^{2}$ \\ James R. Ryan, M.D. ${ }^{3}$ \\ David R. Lucas, M.D. ${ }^{4}$ \\ Jeffrey Muler, M.D. ${ }^{1}$ \\ Keith S. Lanier, M.D. ${ }^{5}$ \\ George Thomas Budd, M.D. ${ }^{6}$ \\ J. Sybil Biermann, M.D. ${ }^{7}$ \\ Frederick J. Meyers M.D. ${ }^{8}$ \\ Karen Antman, M.D. ${ }^{9}$ \\ ${ }^{1}$ Department of Medicine, University of Michigan \\ Medical Center, Ann Arbor, Michigan. \\ ${ }^{2}$ Southwest Oncology Group Statistical Center, \\ Seattle, Washington. \\ ${ }^{3}$ Department of Orthopaedic Surgery, Wayne State \\ University Medical Center, Detroit, Michigan.
}

${ }^{4}$ Department of Pathology, Wayne State University Medical Center, Detroit, Michigan.

${ }^{5}$ Columbia River Community Clinical Oncology Program, Portland, Oregon.

${ }^{6}$ Department of Medicine, Cleveland Clinic Foundation, Cleveland, Ohio.

${ }^{7}$ Department of Orthopaedic Surgery, University of Michigan Medical Center, Ann Arbor, Michigan.

${ }^{8}$ Department of Medicine, University of California-Davis, Sacramento, California.

${ }^{9}$ Department of Medicine, Columbia University, New York, New York.

Supported in part by the following Public Health Service Cooperative Agreement grants awarded by the National Cancer Institute, Department of Health and Human Services: CA38926, CA32102, CA14028, CA45377, CA04919, CA27057, CA46441, CA58882, CA37981, CA76447, CA35178, CA04920, CA45450, CA45807, CA35200, CA42777, CA35262, CA46113, CA20319, CA22433, CA35119, CA58861, and CA67663.

Address for reprints: Southwest Oncology Group (SWOG-9139), Operations Office, 14980 Omicron Drive, San Antonio, TX 78245-3217; E-mail: pubs@SWOG.org

Received September 19, 2003; revision received October 31, 2003; accepted November 14, 2003.
BACKGROUND. The objective of this study was to estimate the time to treatment failure and survival rate of the three-drug combination of doxorubicin, cisplatin, and ifosfamide as primary and postoperative, adjunctive treatment for teenagers and adults with osteosarcoma (OS).

METHODS. Sixty-three eligible patients with nonmetastatic OS of the extremities were registered from 24 institutions from February, 1992 through December, 1996. Chemotherapy was comprised of doxorubicin at a dose of $75 \mathrm{mg} / \mathrm{m}^{2}$ and cisplatin at a dose of $120 \mathrm{mg} / \mathrm{m}^{2}$, alternating with doxorubicin at a dose of $50 \mathrm{mg} / \mathrm{m}^{2}$ and ifosfamide at a dose of $8 \mathrm{~g} / \mathrm{m}^{2}$. Four cycles were given prior to surgical resection, and four cycles were given after surgery. Outcome measures included the time to treatment failure, overall survival, toxicity, and centralized assessment of tumor necrosis.

RESULTS. Thirty-one of 63 eligible patients died, for a 5-year overall survival rate of $58 \%$ (95\% confidence interval $[95 \% \mathrm{CI}], 46-71 \%$ ). The median time to treatment failure was 19 months (95\% CI, 12-41 months). A good pathologic response ( $\geq 90 \%$ necrosis) to neoadjuvant chemotherapy was observed in $48 \%$ of patients who underwent surgery. There was no correlation noted between response to neoadjuvant chemotherapy and patient outcome. Grade 4 hematologic toxicities were frequent $(89 \%)$, although serious nonhematologic toxicities other than nausea and emesis were uncommon.

CONCLUSIONS. The regimen and schedule used in the current study did not improve outcomes compared with prior trials of doxorubicin and cisplatin alone. New, more effective drugs are needed for the treatment of patients with OS. The identification and utilization of molecular markers to predict outcome and response to therapy would facilitate clinical management, limiting exposure to toxic therapies for patients with favorable molecular profiles and identifying those patients who may fail with current approaches as candidates for clinical trials. Cancer 2004;100:818-25. () 2004 American Cancer Society.

KEYWORDS: osteosarcoma (OS), adjuvant chemotherapy, ifosfamide, cisplatin, doxorubicin.

istorically, patients with osteosarcoma (OS) had a dismal prognosis, with retrospective 5-year survival rates of $15-20 \% .^{1,2}$ In 1970 , Marcove et al. reported on 145 consecutive patients, which has become the standard number for a historic control group. ${ }^{2}$ The 5 -year disease-free survival rate in that series was $17.4 \%$, with a time from clinical recognition of metastases to death of approximately 6 months. Since 1978, a number of studies using adjuvant chemotherapy have documented impressive evidence of an increased diseasefree survival rate in patients with primary OS of the extremity, with many reports describing the disease as cured in $\geq 66-75 \%$ of patients afflicted. $^{3-12}$ Agents that are used widely and are accepted as effica- 
cious in patients with OS include doxorubicin, cisplatin, and high-dose methotrexate. Neoadjuvant chemotherapy followed by surgery with additional adjuvant chemotherapy has become a common treatment approach.

Postchemotherapy tumor necrosis has been considered a significant prognostic indicator by many authors. ${ }^{10-15}$ The histologic assessment of response to preoperative chemotherapy requires considerable time, effort, and expertise. ${ }^{16}$ Most attempts to determine response to preoperative chemotherapy by estimating tumor necrosis have been done in single-institution settings. ${ }^{13-15}$ The feasibility and utility of evaluating histopathologic necrosis on a group-wide level at the time of study initiation, however, was uncertain.

Ifosfamide was evaluated in sarcomas in the late 1980s, with reported activity in patients with advanced OS. ${ }^{17,18}$ Based on single-agent activity in patients with soft tissue sarcoma and enhanced response rates in combination with doxorubicin, testing ifosfamide as part of an adjuvant program in OS was deemed appropriate. ${ }^{19,20}$ In 1991, the Southwest Oncology Group (SWOG) initiated a Phase II trial combining ifosfamide with doxorubicin and cisplatin. Based on the perceived primacy of doxorubicin and considering the overlapping renal toxicity of cisplatin and ifosfamide, the trial was designed to deliver doxorubicin for eight cycles in combination with alternating cisplatin and ifosfamide. ${ }^{21}$ The objectives of the current trial were to estimate the survival, time to treatment failure, and toxicity of this approach in teenage and adult populations. Secondary objectives included assessing pathologic necrosis centrally and examining the prognostic utility of percent necrosis after this neoadjuvant chemotherapy regimen.

\section{MATERIALS AND METHODS}

Patients were required to have a histologic diagnosis of OS of the appendicular skeleton, with registration to the protocol occurring within 28 days of biopsy. Allowable stages included high-grade, intracompartmental or extracompartmental disease without clinical evidence of metastasis. Prestudy computed tomography scans of the chest and bone scans were required. Patients had to be age $\geq 11$ years, with no upper age limit specified. Adequate bone marrow reserve (leukocyte count $>3000 / \mu \mathrm{L}$ and platelet count $>$ institutional lower limits), normal renal and hepatic function (serum creatinine and bilirubin levels $<$ institutional upper limits of normal), and left ventricular function ( $>$ institutional lower limits of normal on a prestudy multiple-gated acquisition scan or an echocardiogram) were necessary. A SWOG performance status of $0-2$ was required. Patients were ineligible if they had multicentric primary disease, parosteal OS, periosteal OS, Paget disease, radiation-induced OS, or recurrent disease. No prior chemotherapy or radiation therapy for OS was permitted. Pregnant or nursing women were ineligible, and all participants of reproductive potential agreed to use effective contraception. All patients were aware of the investigational nature of the study and provided Institutional Review Board-approved written informed consent.

\section{Treatment Plan}

Needle biopsy or open biopsy to obtain a diagnosis was accepted. Attention to placement of the biopsy tract, meticulous hemostasis, the use of methyl methacrylate if the bone was entered, and resection of the biopsy tract at the time of definitive surgery were required.

Chemotherapy was comprised of eight cycles, including four preoperative cycles (neoadjuvant chemotherapy) and four cycles after surgery (adjuvant chemotherapy). Courses were to be given at 21-day intervals or on recovery from toxicity from the previous cycle. Chemotherapy was comprised of alternating cycles of doxorubicin/cisplatin and doxorubicin/ ifosfamide. For Cycles 1, 3, 5, and 7, the intended doxorubicin dose was $75 \mathrm{mg} / \mathrm{m}^{2}$ delivered by a 96hour central venous infusion. Cisplatin was given on Day 1 at a dose of $120 \mathrm{mg} / \mathrm{m}^{2}$ in $3 \%$ saline intravenously (i.v.) over 2 hours in odd-numbered cycles. Patients were hydrated prior to cisplatin and received mannitol before and after cisplatin infusion. For $\mathrm{Cy}$ cles $2,4,6$, and 8 , the intended doxorubicin dose was $50 \mathrm{mg} / \mathrm{m}^{2}$ delivered by 96 -hour i.v. infusion. Ifosfamide was given on Days 1-4 of each even-numbered cycle at a dose of $2 \mathrm{~g} / \mathrm{m}^{2}$ over 1 hour. Sodium mercaptoethanesulfonate was given at a dose of 400 $\mathrm{mg} / \mathrm{m}^{2}$ by slow i.v. push at 0 hours, 4 hours, and 8 hours around each daily dose of ifosfamide. The proposed treatment plan resulted in a total doxorubicin dose of $500 \mathrm{mg} / \mathrm{m}^{2}$, a total cisplatin dose of $480 \mathrm{mg} /$ $\mathrm{m}^{2}$, and a total ifosfamide dose of $32 \mathrm{~g} / \mathrm{m}^{2}$. Dose adjustments for severe toxicity were allowed and were made cycle-specific for the subsequent odd or even cycle. For patients who experienced a platelet count $\leq 40,000 / \mu \mathrm{L}$, a leukocyte count $<500 / \mu \mathrm{L}$, or admission to the hospital with neutropenia (neutrophils $<500 / \mu \mathrm{L}$ ) and fever, doses were reduced one level. When doses were reduced for toxicity, they were not increased subsequently. Myeloid growth factors became available during the course of the study, and their use was allowed at the discretion of the treating physician.

Definitive surgery was planned after recovery 
from the Cycle 4 of chemotherapy, approximately on Day 90 of protocol treatment. Margins for resection and location of transmedullary resection were at the discretion of the operating surgeon. Amputation or limb-salvage surgery was acceptable. For patients who underwent limb salvage, margins were required to be disease free in the pathology assessment; if not, then amputation was recommended. Barring complications, postoperative chemotherapy was to be resumed within 14 days of the operation.

\section{Pathology Assessment}

The protocol required submission of the surgical specimen to a central laboratory for scoring the percent necrosis after preoperative chemotherapy. After the removal of excessive soft tissue at the site of surgery, the specimen was cut longitudinally into $5-\mathrm{mm}$ slabs by a band saw and was placed in $15 \%$ formalin. The specimen was shipped overnight to Wayne State University for processing. Slab sections were photocopied, and a grid was drawn on the photocopy to map the locations of the histologic sections. The entire slab was decalcified and processed for histology. Whenever possible, the viable areas and necrotic areas were circled with a fine-tip felt marker, and the areas of each were measured semiquantitatively by placing the slides over graph paper; then, the percent necrosis was calculated. If a surgical specimen was not sent to the central laboratory, then the reported institutional percent necrosis values were included in the results

\section{Preoperative Doxorubicin Dose Intensity}

Preoperative doxorubicin dose intensity (preop dox DI) was recorded for all patients who completed four cycles of neoadjuvant chemotherapy and underwent protocol-directed surgery. The protocol specified $4 \mathrm{cy}-$ cles of neoadjuvant doxorubicin $\left(250 \mathrm{mg} / \mathrm{m}^{2}\right.$ total) delivered in 21-day cycles (84 days). To permit restaging and surgical planning, an additional 7 days were anticipated and were added to the preoperative interval. Preop dox DI for each patient was calculated using the formula preop dox DI $=$ (doxorubicin received/ $\left.\mathrm{m}^{2} / 250 \mathrm{mg} / \mathrm{m}^{2}\right) \times(91$ days/number of days from first treatment to surgery) and was expressed as a percent intended by the protocol.

\section{Statistical Considerations}

Toxicity was evaluated according to standard criteria of the SWOG. ${ }^{22}$ The study design called for 60 eligible patients, with an anticipated annual accrual rate of 20 patients. Survival was measured from the day of registration on study until death from any cause. The time to treatment failure was measured from the day of registration on study until disease progression, re- moval from treatment due to toxicity, refusal, or death from any cause. The distributions of survival and time to treatment failure were estimated by the KaplanMeier method. ${ }^{23}$ Histologic tumor necrosis to chemotherapy, which was scored centrally, was to be used to evaluate response to preoperative treatment. The results presented in this report were based on data that were available September 30, 2002.

\section{RESULTS}

Between February 1992 and December 1996, 65 patients were registered on the study from 24 different institutions. Two patients were ineligible; one patient had an axial primary with lung metastasis, and a second patient had pulmonary metastasis at the time of registration. The median follow-up was 79 months for 32 eligible patients who were last reported alive. Of 63 eligible patients, there were 44 males and 19 females. Ages ranged from 12 years to 70 years, the median age was 22 years, and 15 patients were age $>30$ years. On-study performance status was recorded as 0 in 17 patients (27\%), 1 in 40 patients (63\%), and 2 in 6 patients (10\%). The primary tumor site was the femur in 32 patients, the tibia in 16 patients, the humerus in 6 patients, and other sites in 9 patients. Tumor histology was characterized as OS not otherwise specified in 21 patients, osteoblastic in 19 patients, chondroblastic in 13 patients, fibroblastic in 7 patients, small cell in 2 patients, and telangiectatic in 1 patient. All but six patients had extracompartmental (Enneking Stage IIB) disease. The duration of symptoms prior to diagnosis was recorded as $<1$ month in 7 patients (11\%), $1-6$ months in 42 patients (67\%), and $>6$ months in 14 patients (22\%). Patient and tumor characteristics are summarized in Table 1.

Fifty-seven eligible patients completed 4 cycles of preoperative chemotherapy. One patient was given an incorrect dose of doxorubicin and was taken off treatment because of toxicity. A second patient experienced chest pain after her first cisplatin infusion and refused further therapy. Four additional patients were removed from study during primary chemotherapy for reasons that were not specified in the protocol; two patients were removed for lack of radiologic responses in the primary tumor, one patient was removed because limb salvage was not possible, and one patient moved to a location where an SWOG institution was not available for study continuation.

Fifty-six eligible patients underwent protocol-directed surgery. One patient completed preoperative chemotherapy but refused a recommended amputation and further treatment. Surgery was performed at a median of 103 days (range, 83-148 days) after treatment initiation. Surgical specimens for scoring his- 
TABLE 1

Patient Characteristics

\begin{tabular}{lll}
\hline Characteristic & No. of patients & $\%$ \\
\hline Age (yrs) & & \\
$12-21$ & 29 & 46 \\
$22-30$ & 19 & 30 \\
$31-45$ & 9 & 14 \\
$>45$ & 6 & 10 \\
Gender & & \\
Male & 44 & 70 \\
Female & 19 & 30 \\
Race & & \\
White & 46 & 73 \\
Hispanic & 8 & 13 \\
Black & 6 & 10 \\
Asian & 2 & 3 \\
American Indian & 1 & 2 \\
Site & & \\
Femur & 32 & 51 \\
Tibia & 16 & 10 \\
Humerus & 6 & 14 \\
Other & 9 & \\
\hline
\end{tabular}

topathologic necrosis were received by the central laboratory for 44 patients $(80 \%)$. The median time to resumption of adjuvant chemotherapy after surgery was 16 days (range, 10-41 days).

Postoperative adjuvant chemotherapy was completed by 41 patients. Fifteen patients received less than the planned four courses of adjuvant chemotherapy for the following reasons: toxicity prompted patient and physician decision to stop therapy in 5 patients, refusal to continue by 3 patients, physician decision to change or stop therapy in 4 patients, disease progression in 2 patients, and early death in 1 patient.

\section{Survival}

Of 63 eligible patients, 31 patients had died by the time of last follow-up, for a 5-year overall survival rate of $58 \%$ (95\% confidence interval [95\% CI], 46-71\%) (Fig. 1). The median overall survival was 86 months (95\% CI, 49-92 months). All reported deaths were associated with recurrent OS, including one death on treatment secondary to toxicity. The median time to treatment failure was 19 months $(95 \% \mathrm{CI}, 12-41$ months) (Fig. 2).

Of 63 eligible patients, 32 patients developed recurrent disease. The most frequent site of recurrence was lung $(80 \%)$, although failure occurred locally $(5 \%)$, in bone (10\%), and in soft tissue (3\%). Three patients had distant disease progression during protocol therapy. Each patient who developed recurrent OS either had died or was still alive with disease at the time of last follow-up.

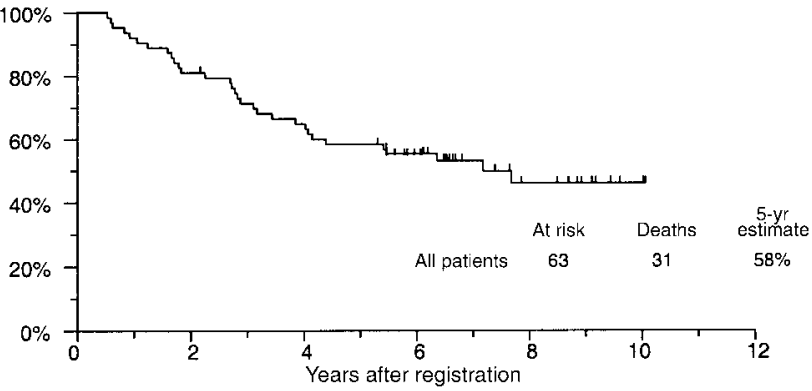

FIGURE 1. Overall survival in eligible patients with follow-up.

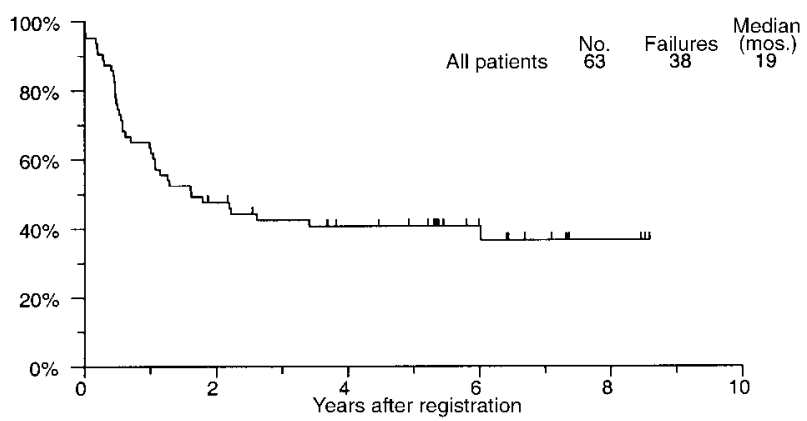

FIGURE 2. The time to treatment failure in eligible patients with follow-up.

Isolated local failure occurred in 3 of 56 patients who underwent protocol-directed surgery. One local failure was in a radius lesion after a poor response to neoadjuvant chemotherapy ( $10 \%$ necrosis) and a 2 -mm soft tissue margin. A second local failure occurred in a femur lesion nearly 6 years after the patient underwent resection (57\% necrosis). The third local failure occurred despite $100 \%$ tumor necrosis in a patient with a fibular OS, small cell variant.

\section{Toxicity}

Of the 63 eligible patients who were evaluable for toxicities during neoadjuvant chemotherapy, 52 patients (83\%) experienced Grade 4 hematologic toxicities. Seven of those patients (11\%) also experienced Grade 4 nonhematologic toxicities, principally gastrointestinal (emesis). Toxicities were similar during postoperative adjuvant chemotherapy. Of 55 evaluable patients, 43 patients (78\%) experienced Grade 4 hematologic toxicity and 5 patients $(9 \%)$ also experienced Grade 4 nonhematologic toxicity. One patient died from infection with associated, severe myelosuppression. Autopsy revealed necrotizing colitis as a likely cause of death; in addition, severe bone marrow hypocellularity and two pulmonary metastatic nodules were found. The worst episodes of toxicity experienced for all eligible patients on study are summarized in Table 2. 
TABLE 2

Toxicity Summary: Worst Toxicity Experienced per Patient $(n=63$ Patients)

\begin{tabular}{lllllll}
\hline & \multicolumn{7}{c}{ Grade } \\
\cline { 2 - 7 } Toxicity & $\mathbf{0}$ & $\mathbf{1}$ & $\mathbf{2}$ & $\mathbf{3}$ & $\mathbf{4}$ & $\mathbf{5}$ \\
\hline Anemia & 8 & 4 & 6 & 28 & 17 & 0 \\
Granulocytopenia & 5 & 0 & 1 & 8 & 49 & 0 \\
Leukopenia & 3 & 0 & 0 & 8 & 52 & 0 \\
Thrombocytopenia & 9 & 3 & 14 & 16 & 21 & 0 \\
Cardiovascular & 55 & 6 & 2 & 0 & 0 & 0 \\
Flu-like symptoms & 13 & 11 & 36 & 2 & 1 & 0 \\
Gastrointestinal & 1 & 13 & 22 & 22 & 5 & 0 \\
Infection & 43 & 0 & 6 & 12 & 1 & 1 \\
Metabolic & 57 & 2 & 2 & 2 & 0 & 0 \\
Mucosal & 17 & 12 & 21 & 9 & 4 & 0 \\
Neuromotor & 54 & 6 & 2 & 1 & 0 & 0 \\
Neurosensory & 36 & 7 & 15 & 5 & 0 & 0 \\
Renal/bladder & 43 & 17 & 1 & 1 & 1 & 0 \\
\hline & & & & & &
\end{tabular}

Hematologic toxicities throughout the study were formidable. Principally, as a consequence of neutropenic fever, i.v. antibiotics were required (usually only once) by 47 patients (75\%). Myeloid growth factor was used in 45 patients $(71 \%)$ for a median of 5 cycles. Growth factor use was more common in patients who had received i.v. antibiotics ( $87 \%$ vs. $44 \%$ ), reflecting common practice at that time. Blood transfusions were required in 34 patients, 15 during neoadjuvant treatment, 8 in the perioperative period, and 17 during postoperative adjuvant chemotherapy. Platelet transfusions were required in 12 patients, 6 during neoadjuvant chemotherapy and 6 during adjuvant treatment. Toxicities contributed to a decision to stop therapy prematurely in at least 11 patients, as described earlier.

Two patients had cisplatin discontinued after Cycles 1 and 3, secondary to tinnitus. There were two patients in whom Grade 3 increases in creatinine levels were reported, both in the setting of additional toxicities. Two elderly patients had asymptomatic decreases in the ejection fraction below institutional normal values, prompting cessation of doxorubicin for Cycles 7 and 8 of therapy; with repeat testing, values for both patients returned to normal. Other cardiac toxicities of $>$ Grade 1 were not observed on study or in follow-up. One middle-aged woman was diagnosed subsequently with early uterine carcinoma and was treated effectively. No secondary myelodysplastic syndromes or leukemias were reported.

\section{Surgery and Pathology Assessment}

Forty-four patients underwent limb-salvage surgery, and 12 patients underwent amputation. Amputation was more common when the primary tumor was in the tibia $(31 \%)$ compared with the femur $(17 \%)$ or other sites (13\%), most likely because of the reconstructive difficulties in the tibia. Pathologic fracture (2 of 4 patients who underwent amputation) and larger primary tumor size (median, $10 \mathrm{~cm}$ for amputation vs. $8 \mathrm{~cm}$ for limb salvage) appeared to influence the likelihood of amputation. Response to neoadjuvant chemotherapy did not appear to influence the surgical procedure performed. Twenty one of 44 patients who underwent limb-salvage surgery had $\geq 90 \%$ necrosis. In the group who underwent amputation, 6 of 11 patients had $\geq 90 \%$ necrosis.

Of 56 patients who underwent protocol-directed surgery, specimens were reviewed by the study pathologist in 44 patients. Amongst those 44 specimens, there was concordance between institution and study pathologists in 41 specimens $(93 \%)$ in assigning a $\mathrm{Hu}-$ vos score $(<90 \%$ or $\geq 90 \%$ necrosis). In 2 specimens, the originating institution described $100 \%$ necrosis and $92 \%$ necrosis; those specimens were characterized subsequently by the study pathologist with $67 \%$ necrosis and $70 \%$ necrosis, respectively. The final specimen was graded with $91 \%$ necrosis by the institution and $100 \%$ necrosis by the study pathologist. When materials were not forwarded to the reference center (12 specimens), the institutional percent necrosis was used in this analysis.

There were 17 patients $(30 \%)$ with $<60 \%$ necrosis, 29 patients $(52 \%)$ with $<90 \%$ necrosis, and 27 patients (48\%) in whom necrosis was estimated at $\geq 90 \%$. Seven patients were assessed with $100 \%$ necrosis, with 5 patients surviving free of disease. When analyzed by the dichotomy of $<90 \%$ or $\geq 90 \%$, there was no significant difference in survival $(P=0.95)$. There was a correlation between preop dox DI and percent necrosis, as described below.

\section{Treatment Received and Preop Dox DI}

The number of chemotherapy cycles and the median doses received for all 63 eligible patients are listed in Table 3. Patients age $\leq 21$ years were more likely to receive 8 cycles of chemotherapy (23 of 29 patients; $79 \%$ ) compared with patients age $>21$ years (18 of 34 patients; 53\%).

The median preop dox DI in 56 evaluable patients was $84 \%$ (range, $61-110 \%$ ). Twenty-three of 56 evaluable patients $(41 \%)$ received $\geq 90 \%$ of the preop dox DI intended by the protocol. Again, preop dox DI was related to age, with patients age $\leq 21$ years receiving a median of $90 \%$ compared with $81 \%$ in older patients and with $54 \%$ of patients age $\leq 21$ years receiving a DI $\geq 90 \%$ compared with $29 \%$ in older patients. A higher preop dox DI also appeared to increase the proportion 
TABLE 3

Treatment Received ( $n=63$ Patients)

\begin{tabular}{lllll}
\hline \multirow{2}{*}{$\begin{array}{l}\text { Maximum } \\
\text { no. of cycles } \\
\text { received }\end{array}$} & $\begin{array}{l}\text { No. of } \\
\text { patients }\end{array}$ & Doxorubicin (mg) & Cisplatin (mg) & Ifosfamide (g) \\
\cline { 3 - 5 } & 3 & 75 & 120 & 0 \\
1 & 3 & - & - & - \\
2 & - & 200 & 240 & 8 \\
3 & 3 & 242 & 240 & 16 \\
4 & 6 & 340 & 16 \\
5 & 3 & 295 & 360 & 22 \\
6 & 3 & 345 & 460 & 22 \\
7 & 4 & 405 & 480 & 32 \\
8 & 41 & 465 & & \\
\hline
\end{tabular}

TABLE 4

Preoperative Doxorubicin Dose Intensity Received Related to Necrosis

\begin{tabular}{llll}
\hline & & \multicolumn{2}{c}{$\begin{array}{l}\text { Fraction of patients who } \\
\text { achieved > 90\% necrosis }\end{array}$} \\
\cline { 3 - 4 } $\begin{array}{llll}\text { Preoperative Dox } \\
\text { DI received }\end{array}$ & $\begin{array}{l}\text { No. of } \\
\text { patients }\end{array}$ & $\%$ & $\mathbf{9 5 \%}$ CI \\
\hline$\langle$ median: $\rangle$ median $^{\mathrm{a}}$ & $28: 28$ & $32: 64$ & $16-52: 44-81$ \\
$\langle 90 \%:\rangle 90 \%$ & $33: 23$ & $36: 65$ & $20-55: 43-84$ \\
\hline
\end{tabular}

Dox: doxorubicin; DI: dose intensity; 95\% CI: 95\% confidence interval.

${ }^{a}$ The median preoperative doxorubicin dose intensity for all 56 evaluable patients was $84 \%$.

of good pathologic responses, as depicted in Table 4 (although the increase was not statistically significant).

Evaluations of survival relative to patient characteristics, tumor variables, and treatment delivered are presented in Tables 5 and 6. Primary site, histology, and preop dox DI appeared to have the most effect on outcome, although a study of this size provides insufficient power to detect differences in survival. Patients who were treated at institutions with more accrual (4 institutions, 35 patients) had improved survival (60\% vs. $50 \%$ ) compared with centers that entered fewer patients (20 institutions, 28 patients).

\section{DISCUSSION}

The triad that includes the patient, the malignancy, and the therapy interacts and determines outcome in a study. This trial showed no apparent improvement despite alternation of a doxorubicin and ifosfamide regimen with the standard doxorubicin and cisplatin. Although 95\% confidence intervals overlapped with previously reported studies, including an earlier SWOG study, recurrence free survival and overall survival rates did not approach the best prior results of a cure rate of $70 \% .^{3-12,24}$

One aspect of the current study that may have
TABLE 5

Patient Characteristics and Tumor Variables Related to Overall Survival

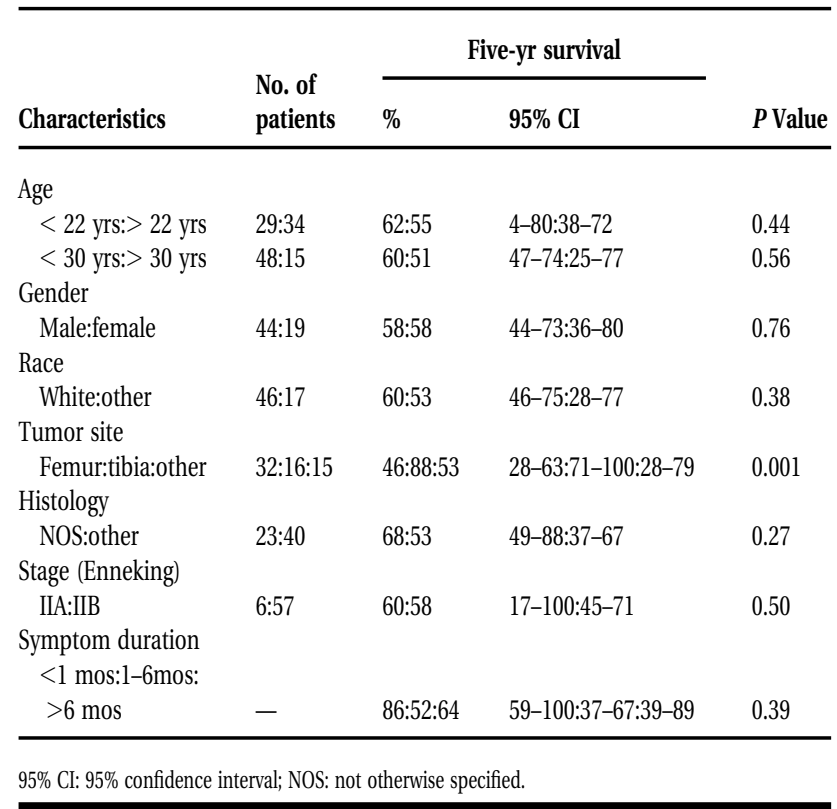

TABLE 6

Patient Treatment Variables Related to Survival from Date of Surgery

\begin{tabular}{|c|c|c|c|c|}
\hline \multirow[b]{2}{*}{ Treatment (n) } & \multirow{2}{*}{$\begin{array}{l}\text { No. of } \\
\text { patients }\end{array}$} & \multicolumn{2}{|c|}{$\begin{array}{l}\text { Five-yr survival from } \\
\text { date of surgery }\end{array}$} & \multirow[b]{2}{*}{$P$ value } \\
\hline & & $\%$ & $95 \% \mathrm{CI}$ & \\
\hline $\begin{array}{l}\text { Limb salvage:amputation } \\
\% \text { Necrosis }\end{array}$ & $44: 12$ & $61: 56$ & $47-76: 26-85$ & 0.95 \\
\hline$<90 \%:>90 \%$ & $29: 27$ & $55: 66$ & $37-73: 47-84$ & 0.76 \\
\hline $\begin{array}{l}\text { Preoperative Dox DI } \\
\text { < Median:> median }\end{array}$ & $28: 28$ & $49: 71$ & $30-67: 55-88$ & 0.31 \\
\hline
\end{tabular}

95\% CI: 95\% confidence interval; Dox: doxorubicin; DI: dose intensity.

contributed to outcome is the proportion of older patients. The median age of study participants was 22 years, and $25 \%$ of those enrolled were age $>30$ years, a known adverse prognostic factor. ${ }^{4,25}$ Although the current study did not include children age $<12$ years, and the power to detect such an effect was limited by the small sample size, the 5-year survival estimate was higher in younger patients. Pediatric populations may have superior outcomes, in part because of increased tolerance of aggressive chemotherapy, as described above. Other factors that conceivably may influence outcomes in older patients include delay in diagnosis and noncompliance with treatment in an adolescent and young adult patient populations.

Adults with OS likely have a different tumor biology with greater heterogeneity compared with chil- 
dren who have this disease. Prognostic factors that have been reported in OS include DNA ploidy; the presence of P-glycoprotein (multidrug resistance); and loss of heterozygosity of the RB locus and, more recently, her2. ${ }^{26-29}$ The biology of the tumors in this and other study populations, however, is largely unknown. Identification and recognition of the impact of these or other variables is one strategy to evaluate results, compare studies, and improve overall outcomes.

Response to preoperative chemotherapy has been considered a prognostic indicator in patients with OS, with a large majority of patients who have $\geq 90 \%$ necrosis at resection surviving recurrence free..$^{8-10,13,25,30}$ This parameter appears to be most predictive in programs that have used high-dose methotrexate. ${ }^{10,11,31}$ In the current study, nearly $50 \%$ of patients achieved a good response, yet a correlation between necrosis and outcome was not clear, and patients who were good responders did not enjoy a similar reported high freedom from recurrence. The longer duration of preoperative treatment and the inclusion of ifosfamide in the preoperative regimen may have increased the proportion of good responders and lessened the utility of scoring necrosis as a prognostic factor, as been observed by others. ${ }^{12,32}$ In the current multiinstitutional study, the concordance of scoring necrosis between study and institutional pathologists was good (93\%). However, processing specimens for shipping interfered with the study pathologist receiving all specimens. Considering the good concordance between pathologists and the lack of a clear correlation between necrosis and outcome, it may not be necessary to assess necrosis centrally in future multiinstitutional studies unless postoperative therapy is dependent on response. Instead, tissue resources and transfers may be utilized better for confirmation of diagnosis and banking for molecular analysis.

Based on the survival of patients without adjuvant chemotherapy, a large majority of patients with OS have micrometastatic disease at the time of diagnosis. Survival is related to the burden of micrometastatic disease, the presence of nonpulmonary metastasis, and the chemosensitivity of disease. The numbers of patients who progressed in the current study during primary chemotherapy or study treatment were few, and the fraction of good responders to primary chemotherapy did not differ obviously from other studies. ${ }^{4,8,9,11,12}$ A long median time to recurrence and the inability to salvage patients who developed recurrent disease suggest a larger tumor burden, an increase in extrapulmonary micrometastatic disease sites, and a slower growth fraction with less inherent sensitivity to chemotherapy.

Treatment variables include agents used as well as dose and schedule, duration of therapy, timing and adequacy of surgery, and DI received. Doxorubicin and cisplatin are cornerstones of most modern OS regimens. ${ }^{5,6,8,9,12,33}$ The protocol dose and schedule of these agents in the current study were comparable to those of other regimens. ${ }^{5,8,9,12,33}$ However, the dose rate of cisplatin was lower because of the alternating therapy. Ifosfamide has been used increasingly with reported efficacy. ${ }^{32-35}$ High-dose methotrexate was not included in the current study, and the impact of its exclusion is not known. However, in randomized trials of high-dose methotrexate, an advantage for this approach has not been established. ${ }^{8,15,36-38}$ There was no attempt at postoperative tailoring of chemotherapy in this trial, although the value of that approach has not been demonstrated clearly. ${ }^{9,11}$ The total duration of treatment was shorter than some programs, but toxicity and tolerance were issues in many patients, and completing the study treatment as outlined was difficult. Local failure was uncommon (5\%); and, although delays in surgery to 90 days may have increased the percent of good responders to primary chemotherapy artificially, nothing suggests that the timing of surgery compromised overall outcomes. The DI received also was comparable to previous reports and had no apparent influence on outcome. ${ }^{21,39}$

Although combination chemotherapy clearly appears to improve outcomes for patients with OS, the value of continuing efforts at increasing DI or adding nonspecific cytotoxic agents seems limited. Instead, identification and utilization of molecular markers that are prognostic and predictive of outcome and response to therapy are needed. Future clinical research efforts should emphasize limiting exposure to toxic and difficult therapies in patients with favorable molecular profiles and developing targeted, specific therapies for patients who are destined for failure with current approaches.

\section{REFERENCES}

1. Friedman MA, Carter SK. The therapy of osteogenic sarcoma. Current status and thoughts for future. J Surg Oncol. 1972;4:482-510.

2. Marcove RC, Mike V, Hajek JV, et al. Osteogenic sarcoma under the age of twenty-one. J Bone Joint Surg. 1970;52A: 411-422.

3. Link MP, Goorin AM, Miser AW, et al. The effect of adjuvant chemotherapy on relapse-free survival in patients with osteosarcoma of the extremity. N Engl J Med. 1986;314:1600-1606.

4. Meyers PA, Heller G, Healey J, et al. Chemotherapy for nonmetastatic osteogenic sarcoma: the Memorial SloanKettering experience. J Clin Oncol. 1992;10:5-15.

5. Bacci G, Ferrari S, Bertoni F, et al. Long-term outcome for patients with nonmetastatic osteosarcoma of the extremity treated at the Istituto Ortopedico Rizzoli according to the Istituto Ortopedico Rizzoli/osteosarcoma-2 protocol: an updated report. J Clin Oncol. 2000;18:4016-4027. 
6. Arndt CAS, Crist WM. Common musculoskeletal tumors of childhood and adolescence. N Engl J Med. 1999;341:342-352.

7. Link MP, Goorin AM, Horowitz M, et al. Adjuvant chemotherapy of high-grade osteosarcoma of the extremity. Clin Orthop. 1991;270:8-14.

8. Bramwell VHC, Burgers M, Sneath R, et al. A comparison of two short intensive adjuvant chemotherapy regimens in operable osteosarcoma of limbs in children and young adults: the first study of the European Osteosarcoma Intergroup. J Clin Oncol. 1992;10:1579-1591.

9. Winkler K, Beron G, Delling G, et al. Neoadjuvant chemotherapy of osteosarcoma: results of a randomized cooperative trial (COSS-82) with salvage chemotherapy based on histological tumor response. J Clin Oncol. 1988;6:329-337.

10. Glasser DB, Lane JM, Huvos AG, et al. Survival, prognosis, and therapeutic response in osteogenic sarcoma: the Memorial Hospital experience. Cancer. 1992;69:698-708.

11. Provisor AJ, Ettinger LJ, Nachman JB, et al. Treatment of nonmetastatic osteosarcoma of the extremity with preoperative and postoperative chemotherapy: a report from the Children's Cancer Group. J Clin Oncol. 1997;15:76-84.

12. Meyers PA, Garlick R, Heller G, et al. Intensification of preoperative chemotherapy for osteogenic sarcoma: results of the Memorial Sloan-Kettering (T12) protocol. J Clin Oncol. 1998;16:2452-2459.

13. Rosen G, Capparros B, Huvos M, et al. Pre-operative chemotherapy for osteosarcoma: selection of postoperative adjuvant chemotherapy base on the response of the primary tumor to preoperative chemotherapy. Cancer. 1982;49:1221-1230.

14. Ward WG, Mikaelian K, Dorey F, et al. Pulmonary metastases of Stage IIB extremity osteosarcoma and subsequent pulmonary metastases. J Clin Oncol. 1994;12:1849-1858.

15. Hudson M, Jaffe MR, Jaffe N, et al. Pediatric osteosarcoma: therapeutic strategies, results, and prognostic factors derived from a 10-year experience. J Clin Oncol. 1990;8:1988-1997.

16. Raymond AK. Osteosarcoma: specimen management after preoperative chemotherapy and arteriogram-directed dissection. Presented at Optimizing Management of Primary Bone Tumors: An International Symposium Emphasizing the Multidisciplinary Approach, Houston Texas, November 2-5, 1988.

17. Marti C, Kroner T, Remagen W. High-dose ifosfamide in advanced osteosarcoma. Cancer Treat Rep. 1985;69:115-117.

18. Pratt CB, Horowitz ME, Meyer WH, et al. Phase II trial of ifosfamide in children with malignant solid tumors. Cancer Treat Rep. 1987;71:131-135.

19. Antman KH, Ryan L, Elias A, et al. Response to ifosfamide and mesna: 124 previously treated patients with metastatic or unresectable sarcoma. J Clin Oncol. 1989;7:126-131.

20. Elias A, Ryan L, Sulkes A, et al. Response to mesna, doxorubicin, ifosfamide, and dacarbazine in 108 patients with metastatic or unresectable sarcoma and no prior chemotherapy. J Clin Oncol. 1989;7:1208-1216.

21. Smith MA, Ungerleider RS, Horowitz ME, et al. Influence of doxorubicin dose intensity on response and outcome for patients with osteogenic sarcoma and Ewing's sarcoma. J Natl Cancer Inst. 1991;83:1460-1470.

22. Green S, Weiss GR. Southwest Oncology Group standard response criteria, endpoint definitions and toxicity criteria. Invest New Drugs. 1992;10:239-253.

23. Kaplan EL, Meier R. Nonparametric estimation from incomplete observation. J Am Stat Assoc. 1958;53:457-481.
24. Sutow WW, Gehan EA, Dyment PG, et al. Multidrug adjuvant chemotherapy for osteosarcoma: interim report of the Southwest Oncology Group Studies. Cancer Treat Rep. 1978; 62:265-269.

25. Bielack SS, Kempf-Bielack B, Delling G, et al. Prognostic factors in high-grade osteosarcoma of the extremities of trunk: an analysis of 1,702 patients treated on neoadjuvant cooperative osteosarcoma study group protocols. J Clin Oncol. 2002;20:776-790.

26. Look AT, Douglass EC, Meyer WH. Clinical importance of near-diploid tumor stem lines in patients with osteosarcoma of an extremity. N Engl J Med. 1988;318:1567-1572.

27. Chan HSL, Grogan TM, Gaddad G, et al. P-glycoprotein expression: critical determinant in the response to osteosarcoma chemotherapy. J Natl Cancer Inst. 1997;89:1706-1715.

28. Feugeas O, Guriec N, Babin-Boilletot A, et al. Loss of heterozygosity of the RB gene is a poor prognostic factor in patients with osteosarcoma. J Clin Oncol. 1996;14:467-472.

29. Gorlick R, Huvos AG, Heller G, et al. Expression of HER2/ erbB-2 correlates with survival in osteosarcoma. J Clin Oncol. 1999;17:2781-2788.

30. Davis AM, Bell RS, Goodwin PJ. Prognostic factors in osteosarcoma: a critical review. J Clin Oncol. 1994;12:423-431.

31. Bacci G, Ferrari S, Delepine N, et al. Predictive factors of histologic response to primary chemotherapy in osteosarcoma of the extremity: study of 272 patients preoperatively treated with high-dose methotrexate, doxorubicin, and cisplatin. J Clin Oncol. 1998;16:658-663.

32. Bacci G, Briccoli A, Ferrari S, et al. Neoadjuvant chemotherapy for osteosarcoma of the extremity: long-term results of the Rizzoli's 4th protocol. Eur J Cancer. 2001;37:2030--039.

33. Meyers P, Schwartz C, Bernstein M, et al. Addition of ifosfamide and muramyl tripeptide to cisplatin, doxorubicin and high-dose methotrexate improves event free survival (EFS) in localized osteosarcoma. Proc Am Soc Clin Oncol. 2001;20: 1463.

34. Meyer WH, Pratt CB, Poquette CA, et al. Carboplatin/ifosfamide window therapy for osteosarcoma: results of the St Jude Children's Research Hospital OS-91 trial. J Clin Oncol. 2001;19:171-182.

35. Harris MB, Cantor AB, Goorin AM, et al. Treatment of osteosarcoma with ifosfamide: comparison of response in pediatric patients with recurrent disease versus patients previously untreated: a Pediatric Oncology Group study. Med Pediatr Oncol. 1995;24:87-92.

36. Souhami RL, Craft AW, Van der Eijken JW, et al. Randomised trial of two regimens of chemotherapy in operable osteosarcoma: a study of the European Osteosarcoma Intergroup. Lancet. 1997;350:911-917.

37. Cortes E, Necheles TF, Holland JF, et al. Adjuvant therapy of operable primary osteosarcoma: a Cancer and Leukemia Group B experience, In: Salmon S, Jones S, editors. Adjuvant therapy of cancer. Volume 3. New York: Grune \& Stratton, 1981:201-210.

38. Krailo M, Ertel E, Makley J, et al. A randomized trial comparing high dose methotrexate with moderate dose methotrexate as components of adjuvant chemotherapy in childhood nonmetastatic osteosarcoma. Med Pediatr Oncol. 1987;15:69-77.

39. Lewis IJ, Weeden S, Machin D, et al. Received dose and doseintensity of chemotherapy and outcome in nonmetastatic extremity osteosarcoma. J Clin Oncol. 2000;18:4028-4037. 